\title{
Cannabis (Marijuana)
}

\begin{abstract}
Cannabis Sativa is the most consumed drug in the world next to alcohol. This article describes its effects, especially that of $\Delta^{9}$-THC. The perception of the risk of smoking Cannabis has followed a downward curve in the last 15years Granting it to connotation of "recreational" and ignoring or ignoring its risks for psychophysical health. In some countries of the Northern Hemisphere it is usually combined with phencyclidine, being called the mixture "super yerba". In Argentina, until now, it is considered an unauthorized drug for commercialization, and it comes from Paraguay through drug trafficking. It is article contains the symptoms produced by your toxicokinetics and toxicity, as well as the reference of your treatment. This article also provides the therapeutic effects of CBD, the latter always being used as a second line medication (Cannabis Oil). ${ }^{1}$
\end{abstract}

Keywords: $\Delta^{9}$ THC-CBD, toxicity, health, therapeutic effects, dogga

This drug or its products also receive other names besides marijuana: hashish or hashish, bhang, ganja, dogga

Popular names: Grass, hashish or hashish, yerba mate, smoke, porro, dry (cigarette smoke), weed.

It is the hemp of India, annual herbaceous plant, of Central Asian origin, belonging to the family of cannabáceas with one species: cannabis sativa and two varieties: Indica and American. All the plants of the cannabáceas family are dioicas: they have small masculine and feminine flowers in different plants. Those flowers are also called "buds". It is a drug that has its own entity, since it cannot be incorporated into classic classifications of A) Central Nervous System Depressant Drugs (CNS), B) Stimulant Drugs of the CNS and C) Hallucinogenic Drugs, because it has all three properties. The cannabis sativa (grown) as well as being cultivated drug abuse, also for fiber production and in this case the plant has a low concentration $(<0.25 \%)$ of $\Delta^{9}$ - tetrahydrocannabinol (THC $\Delta^{9)},{ }^{2-5}$ and cannabidiol discharge (CBD), the first of the psychoactive effects being responsible, which in this case is minimal or negligible. ${ }^{6}$

It is the drug of illicit commercialization whose consumption is the most spread in the West. In our country, its consumption occupies the third place of drugs of abuse, after alcohol. Recent studies (October 2016) of the SEDRONAR, report that in the last 10years the consumption of marijuana tripled. The perception of the risk of smoking marijuana has followed a downward curve in the last $15 y e a r s$, giving it a connotation of "recreational use" and ignoring or ignoring its risks for psychophysical health. In some countries of the northern hemisphere it is usually combined with phencyclidine, being called the mixture "superyerba". This combination causes more intense hallucinations or illusions and has greater toxicity than marijuana as monodrug. ${ }^{7-11}$

\section{History}

Its use for recreational and medicinal purposes has been known since antiquity, appearing in documents of the Chinese dynasty of Nung, year 2327a.C. Also in Greece, Herodotus already had knowledge of her. Its therapeutic use was spread in the mid-nineteenth century as an appetite stimulant, sedative, analgesic, antiparasitic, antidiarrheal, bronchodilator, lactogenic, anti-migraine, ${ }^{12}$ and as a treatment of other pathologies: memory loss, tetanus, dysmenorrhea,
Volume 6 Issue 2 - 2019

\author{
Norma EVallejo \\ Professor Emérita Faculty of Medicine, University of Buenos \\ Aires (UBA), Argentina
}

Correspondence: Norma EVallejo, Professor Emérita Faculty of Medicine, University of Buenos Aires (UBA), Argentina, Email vallejonormaelena@hotmail.com

Received: February 14, 2019 | Published: March 21, 2019 gonorrhea, psychosis post childbirth and opium addiction. ${ }^{13}$ At the end of the $19^{\text {th }}$ century, the cannabis was replaced by more effective drugs. ${ }^{14,15}$ Currently some health professionals recommend its use for the treatment of nausea and vomiting caused by antineoplastic chemotherapy, glaucoma for its effect of reducing intraocular pressure, asthma, anxiety, pain and multiple sclerosis. These applications are not currently universally accepted in the medical field, since there are other drugs of proven efficacy and that do not have the psychoactive effects and potential abuse of marijuana. Based on this, the Researchers and the Medical Laboratories are working on the possible therapeutic effects of the CBD and not on the $\Delta^{9}$-THC. In some countries or states, according to current legislation, this type of medication is authorized or not.

\section{Botanical aspects}

The plant is 2 to $5 \mathrm{~m}$ tall and grows in humid geographic areas. Its stem has a length of up to $3 \mathrm{~m}$, is erect and strong and is covered with hairs. The leaves, which are opposite, are located in the lower $3 / 4$ parts of the stem. The upper ones are smaller, with jagged edges and emerge alternately from the stem. The flowers are small, yellow or green and are distributed at the apex of the stem. The plant is dioica. The female is composed of a single floral envelope, around the ovary, which contains a single ovule. The masculine ones are grouped in clusters. The fruit is light green or pale brown, with an ellipsoidal shape up to $5 \mathrm{~cm}$ in length. It has an aromatic odor and spicy flavor and contains only one seed. All parts of plants, whether male or female, possess the psychoactive substances that are called cannabinoids. The concentration of $\Delta^{9}$ tetrahydrocannabinol $\left(\Delta^{9}-\mathrm{THC}\right)$ in it, 20years ago and more, varied between 0.5 and $11 \%$, with its highest concentrations in the flowered heads, located at the ends of the plant. Currently by botanical procedures the concentration can rise to $15-20 \%$ in dry and fresh $20-30 \%$. This variety of cannabis sativa is popularly known as "super marijuana". ${ }^{16,17}$

The dry resinous exudate of these capes is called hashish or hashish in the Middle East and North Africa. In the Far East it is called charas and it is the one that contains the highest amount of $\Delta^{9}$ THC. The hash oil is a dark liquid that contains $20-30 \%$ more than $\Delta^{9}$-THC. The seeds that contain the embryos of the flowers have a slightly lower potency. It is called seedless to the top flowered, seedless female plant. It is called bhang to the dry leaves and buds in flower of the plant (those that contain minor amount of active principle). It receives the name of ganja the resinous mass of the smallest leaves and groups 
of inflorescences. In general, at present, the term marijuana is used to refer to any part of the plant or extract thereof that produces somatic or psychic changes in man. The most common is that the plant is cut, dried, chopped and incorporated into cigarettes. The vegetable contains more than 400chemical compounds, 60 or more of which are cannabinoids. The three most abundant are cannabinol (CBN), cannabidiol (CBD), and several isomers of tetrahydrocannabinol. ${ }^{18}$

Delta 9 tetrahydrocannabinol $\left(\Delta^{9}-\mathrm{THC}\right)$ is the isomer responsible for most of the psychoactive effects of marijuana.

Cannabinol is a metabolite produced by the oxidation of $\Delta^{9}$-THC in plants stored for prolonged periods, and also in the aged hachis. The $\Delta^{8} \mathrm{THC}$ It exists in minimum quantities in the plant and its effects are similar to those of $\Delta^{9}$-THC. While most other cannabinoids are not psychoactive, they can interact with $\Delta^{9}$-THC, increasing or decreasing their potency. ${ }^{19}$ Regarding the classification between the two types of plants mentioned, the one used as a drug of abuse for its richness in $\Delta^{9}$-THC and the one that is produced for fiber, usually uses an approximation method that classifies the plant as a "drug" phenotype or "fiber" according to the following formula:

$$
\text { Phenotype }=\frac{(\%(9-\mathrm{THC}+\% \mathrm{CBN})}{\% C B D}
$$

If the result is greater than 1 , it is considered a "drug" phenotype and if it is less than 1, a "fiber" phenotype. ${ }^{6}$

\section{Properties and mechanisms of action}

The cannabinoids are very liposoluble and produce alterations in the permeability of the membranes, similar to those produced by the very lipophilic general anesthetics. A cannabinoid system is currently recognized constituted by:

\section{Specific cannabinoid receptors: $\mathrm{CB}$ I and CB2}

The $\mathrm{CB} 1$ receptor is the most important in the brain. It is preferably located in the SNC and peripheral; also in the retina. The $\mathrm{CB} 2$ receptors are located in the immune system and in the retina. The CB1 receptor belongs to the family of G-protein-linked receptors. This receptor is linked to an inhibitory G protein (G1), which in turn is linked to adenyl cyclase in an inhibitory manner. It is found in large concentrations in the basal ganglia, hippocampus, cerebellum and cerebral cortex.

\section{Endogenous ligands of the receptors CBI and CB2}

Anandamide: derived from arachidonic acid, is a ligand of the $\mathrm{CB} 1$ receptor located in the CNS.

Palmitoylethanolamine: Is a ligand of a receptor similar to $\mathrm{CB} 2$ located in SNP.

2-Araquidonilglicerol is found in the brain in higher concentrations than anandamide, although the latter is the one that has been most studied. Both the Anandamide such as 2-arachidonylglycerol are mixed $\mathrm{CB} 1 / \mathrm{CB} 2$ agonists.

Currently there is information that there is other $\mathrm{CB}$ receivers. ${ }^{20}$

\section{Final process of transmission of the biological signal}

It consists of the completion of the activation of the cannabinoid receptors for their endogenous ligands. For this, they have to be recapped by a transport system at the level of the plasma membrane. This transporter has not been identified to date and in the in case of not existing, the transport would be done by simple diffusion. Already within the cell, anandamide is degraded by the action of a hydrolase specific for fatty acid amides, called FAAH. Anandamide is inactivated by hydrolysis in 5-20minutes by the action of this amidohydrolase. Many cannabinoid receptors are found in areas of the brain that regulate judgment, pleasure, memory, thinking, concentration, learning, and sensory and time perception. Also the coordination of the movement. The $\Delta^{9}$-THC interacts with the noradrenergic, dopaminergic, serotonergic, cholinergic and GAB Aergic neurotransmitter systems.

Small doses of $\Delta^{9}$-THC produce changes in the CNS that is specific to cannabinoids, while higher doses cause both these specific effects and those that characterize other hypnosedating drugs. The acute administration of cannabis increases the release of dopamine in the nucleus accumbens and a functional interaction between the cannabinoid and opioid systems in the addictive behavior has been described. Also in the cortical mesolimbic pathway the release of dopamine induced by $\Delta^{9}$-THC is carried out through the indirect activation of a $\mu$ receptor located in the ventral tegmental area of the brain, activating the brain reward circuit for drugs of abuse and other reinforcers. ${ }^{21}$ This explains how this system is involved in the process of addiction (reinforcement/dependence/abstinence) to other drugs of abuse (opioids, alcohol, cocaine, amphetamines, nicotine, volatile solvents). Below is reproduced, with permission, part of the publication Number 02-3859 (S) August 2005, of the National Institute on Drugs Abuse (NIDA) of the United States that specifies the brain regions and their functions affected by the use of marijuana and which is still in force today (Table 1). ${ }^{22}$

\section{Endocannabinoides}

S on endogenous substances related to arachidonic acid, discovered descubieros inferred from the sites of action of cannabis on the brain and periphery. They are retriggered neurotransmitters, multifunctional with various actions. The anandamine it is a ligand of the CB1 receptor located in the CNS (Central Nervous System) and the plmitoi letanolamide is a ligand of a receptor similar to CB2 located in S NP. (Peripheral nervous system). The consumption of cannabis induces a growth in the activity of the limbic system, directly stimulating the pleasure and reward mechanisms of the brain. ${ }^{23}$

\section{Toxicokinetics}

The main route of use is inhalation through smoking. In this way, the highest percentage of bioavailability of $\Delta^{9}$-THC is obtained (between 10 to $50 \%$ of this active principle); on the other hand, through the digestive tract, in this case mixed with foods and / or biscuits, absorption are significantly lower (between 1 and $10 \%$ ). The maximum concentrations of $\Delta^{9}$-THC in plasma are reached between 7 and 8 minutes after starting smoking, then declining. By mouth this peak is at $45^{\prime}$. In habitual smokers, through the use of a more effective technique, a higher plasma concentration is observed than in occasional consumers. The ${ }^{9}$-THC is rapidly absorbed by the pulmonary route. It only takes 15 seconds for the lungs to absorb the THC and it is transported to the brain, beginning its action at 5-10minutes. ${ }^{24}$

It has a very short period of semi - elimination in plasma where it is almost totally bound to proteins (99\%). It is then distributed in the liver and peripheral tissues with a high lipid content. This wide distribution in the lipoid tissues is responsible for a large volume of distribution $\left(101 / \mathrm{Kg}\right.$.) Although peaks of $\Delta^{9}$-THC in plasma are 
reached within a few minutes of starting smoking, the maximum clinical effects (cardiovascular and CNS) appear only at 10-30' and may last from $1-4 \mathrm{~h}$, when the consumption is by inhalation. When oral, the clinical manifestations appear at 2-3h. This is because the drug must be distributed from the plasma compartment to the brain and other tissues. Oral absorption increases in a fatty medium. There is no close correlation between blood concentrations and psychosomatic effects, since these correlate better with the total of $\Delta^{9}-\mathrm{THC}$ in Free State only. ${ }^{25}$

Table I Brain regions and their functions

\begin{tabular}{|c|c|}
\hline Brain region & Functions associated with that region \\
\hline \multicolumn{2}{|c|}{ Regions of the brain in which cannabinoid receptors are abundant } \\
\hline Cerebellum & Coordination of body movements \\
\hline Hippocampus & Learning and memory \\
\hline $\begin{array}{l}\text { Cerebral cortex, especially the cingulate, frontal and } \\
\text { parietal regions }\end{array}$ & Superior cognitive functions \\
\hline Nucleus accumbens & Gratification \\
\hline Basal ganglia & Movement control \\
\hline \multicolumn{2}{|l|}{ Reticulated black substance } \\
\hline \multicolumn{2}{|l|}{ Entopeduncular nucleus } \\
\hline \multicolumn{2}{|l|}{ Globe pale (globus pallidus) } \\
\hline \multicolumn{2}{|l|}{ Putamen } \\
\hline \multicolumn{2}{|c|}{ Regions of the brain where there is a moderate concentration of cannabinoid receptors } \\
\hline Hypothalamus & $\begin{array}{l}\text { Bodily disposition functions (temperature regulation, salt and water } \\
\text { balance, reproductive function) }\end{array}$ \\
\hline Brain tonsil & Emotional response, fear \\
\hline Spinal cord & Peripheral sensations, including pain \\
\hline Stem of the brain & Sleep and wakefulness, temperature regulation, motor control \\
\hline Central gray substance & Analgesia \\
\hline Anucleus of the tract solitary algesia & Visceral sensation, nausea and vomiting \\
\hline
\end{tabular}

By smoking continuously, plasma concentrations may be greater than $200 \mathrm{ng} / \mathrm{ml}$. The $\Delta^{9}-\mathrm{THC}$ is then metabolized slowly in tissues and excreted with a final half-life of $18-48 \mathrm{~h}$. Approximately $65 \%$ of the metabolites are excreted in the faeces and 18 to $23 \%$ in the urine, eliminating slowly in 50 days between $80 \%$ and $90 \%$ of a certain amount of drug. Threshold dose of $\Delta^{9}$-THC (the minimum dose is capable of producing euphoria) $50 \mu \mathrm{g} / \mathrm{Kg}$ (WHO). This represents the dose of $3.5 \mathrm{mg}$ for a person of $70 \mathrm{Kg}$. It should be noted that only $50 \%$ of the $\Delta^{9}$-THC content in cannabis. It is absorbed by the human body when it is smoked. The Cannabis Seed Dose is unknown, but it is estimated that it can be approximately $30 \mathrm{mg} / \mathrm{Kg} .{ }^{26}$

Low doses: 3-4mg produces euphoria, mild sedation and disorientation and disinhibition.

High doses: $15 \mathrm{mg}$ produce orthostatic hypotension, tachycardia, obtundation, anxiety, CNS depression and psychotic symptoms. Doses of $100 \mu \mathrm{g} / \mathrm{Kg}$ of THC produce perceptive and sensory alterations.

Hallucinogenic doses: $200-250 \mu \mathrm{g} / \mathrm{Kg}$ via pulmonary (per smoked).

The weight of 1 marijuana cigarette is variable and can range between $250 \mathrm{mg}$ of the plant and $1 \mathrm{~g}$. The smoking of 1 marijuana cigarette affects fine motor coordination, interfering in the handling of motor vehicles. In our country, the most frequently used joints weigh between $500 \mathrm{mg}$ and $800 \mathrm{mg}$. The concentration of $\Delta^{9}$-THC is also variable, depending on the richness of the same as the plant used, and can reach the so-called "super marijuana" up to about $30 \%{ }^{27}$

The slow elimination (sometimes of 4 to 6 weeks) is attributed to:

a. The high solubility in lipids.

b. The enterohepatic circulation.

c. The deep distribution in fatty tissues.

d. The renal reabsorption.

The $\Delta^{9}$-THC is rapidly metabolized and almost entirely in the liver, through the microsomal enzymes that hydrolyze $\Delta^{9}$-THC. Only less than $1 \%$ is eliminated through urine without metabolism.

\section{Its two most important metabolites are II-hydroxy- THC (active) and 9-carboxy THC.}

The first one, is in insufficient concentrations to produce clinical effect when marijuana is smoked, but orally, as the $\Delta^{9}$-THC has poor absorption and is strongly metabolized during its first passage through the liver, the relative increase in the Circulation of 11- hydroxy THC may produce some effects. Large smokers remove ${ }^{9}$-THC from plasma more rapidly (20 to $30 \mathrm{~h}$ ) than non-users $(25$ to $60 \mathrm{~h})$. The $\Delta^{9}$-THC and its degradation products have transplacental and mammary passage. 


\section{Toxicodynamics}

Tolerance it develops rapidly for most purposes, mainly the autonomous and psychoactive. This tolerance is fundamentally of toxicodynamic cause due to the decrease in the activity of CB1 receptors. It has crossed tolerance with alcohol and opiates, most of the central and peripheral effects of cannabis develop tolerance when administration lasts for several days. In laboratory animals it is seen in a range of 3-7days, for analgesia, hypothermia and motor inhibition. The chronic activation of cannabinoids activates the opioid system in brain areas related to motor functions and cognitive and emotional responses, and in neuroendocrine control, which can generate adaptive neuronal phenomena.

\section{Tolerance and dependence tend to develop concomitantly}

Dependence and addiction are currently recognized, although the withdrawal syndrome caused by the suppression or abrupt reduction of the dose of consumption is not serious. The actual addiction consists of the appearance of "craving" and the consequent uncontrollable search for the substance. ${ }^{28}$

\section{Signs and symptoms of acute intoxication}

According to the Author's experience, of the total Emergency Surveys for Abuse Drugs a Sevice Guard Hospital Hospitals Buenos Aires City is not frequent reception of patients intoxicated with marijuana by smoking, with the exception of the "testers". This is due to the following factors:

a. Self-regulation of the dose consumed.

b. Low level of alarm and minimization of risks with social acceptance by part of the consumer and his environment

c. The clinical-psychiatric repercussion of the drug, in general, does not produce extreme alarm reactions that make us suspect critical situations that endanger the life of the patient.

It should be kept in mind that at the level of Clinical Toxicology and Emergency Medicine, the appearance of signs and symptoms almost immediate to the inhalation of the smoke of a marijuana cigarette can be detected, which can be defined as

\section{Early signs and symptoms}

a. Bilateral conjunctival injection

b. Mydriasis (less frequently transient miosis or normal pupils)

c. Photophobia

d. Increase in appetite

e. Dry mouth mucosa

f. Oropharyngitis

g. Uvular edema

h. Thirst

i. Rhinitis

j. Tachycardia

k. Incipient ataxia

It was already mentioned that the maximum peak of its effects usually occurs between $10^{\prime} 30$ 'and usually lasts from 4 to 8 hours.
Consumption orally causes different manifestations in terms of the time of onset of the effects, which are more deferred and prolonged due to their metabolic step and also, in general, at equal doses, less intense and different. It should be noted that thirst may motivate the consumer of marijuana to consume alcoholic beverages, predominantly beer and / or "energizing " beverages and manifest in the doctor's office an association of symptoms combined and/or intensified by the interactions between them. ${ }^{28,29} \mathrm{It}$ is also common the association of marijuana with the consumption of other psychoactive drugs, such as alcohol, cocaine and synthetic drugs.

\section{Hyperemesis syndrome cannabinoid 20}

It can appear later and requires diagnosis, treatment, observation and control of the evolution due to disorders of the Internal Environment with alteration of the Acid-Base Balance and Acute Renal Failure. This syndrome usually manifests episodically in chronic users and occurs repeatedly, but separated by weeks or months between episodes.

The clinical picture of acute poisoning is dose-dependent. It can be presented as "florida" clinical form or as "incomplete clinical form". The following signs and symptoms can be observed, depending on the dose received, presence or absence of tolerance and individual response, particularly in the "testers", who do not have tolerance installed

1. Relaxation and well-being

2. Euphoria (for acting on the system of reward or gratification of the brain, stimulating the brain cells to produce the release of dopamine)

3. Dysphoria

4. Apathy

5. Mydriasis/Miosis/Normal pupils

6. Conjunctival injection

7. Nistagmus

8. Commitment of the visual field

9. Eyelid ptosis

10. Amimia

11. Dry mucous membranes (particularly the mouth with thirst)

12. Inhibition of sweating

13. Trismus

14. Fine tremor

15. Constipation

16. Pruritus

17. Urticaria

18. Exanthema

19. Pallor

20. Cold hands

21. Sleep or depression after the euphoria

22. Hypothermia (usually in children due to accidental ingestion) 
23. Hyperthermia

24. Increase in appétit

25. Pharyngitis

26. Rhinitis

27. Pneumonitis

28. Neumediastine (secondary to deep inhalation due to excessive alveolar distention and alveolar rupture)

29. Commitment of cognitive functions

30. Altered temporo- spatial perception

31. Altered perception (increased perception of visual, auditory, tactile, gustatory and olfactory stimuli)

32. Compromise of skill and motor skill

33. Disorders in gait (ataxia) and balance

34. Vertigo

35. Word dragged

36. Urinary retention

37. Sinus tachycardia

38. Hypertension of the systolic pressure (Increase of the systolic pressure in the supine position).

39. Orthostatic hypotension (decrease in systolic blood pressure in a standing position)

40. Flashback (rare)

41. Immotivated laughs

42. Depersonalization

43. Anxiety

44. Agitation

45. Anxiety crisis

46. Fear

47. Panic attacks

48. Obsessive thoughts

49. Decrease in short-term memory

50. Dispersion of attention, disorders of judgment and other cognitive functions

51. Confusion

52. Illusion - Hallucinations

53. Erratic behavior

\section{Loss of body image}

Acute Toxic Psychosis (more frequently with high doses or in inexperienced users), although it should be kept in mind that in patients with genotypes for schizophrenia, the consumption of a first dose, still relatively low or moderate, can trigger schizophrenic psychosis, requiring treatment of this pathology. Hallucinations, illusions and agitation, delirium and depersonalization appear.
Regarding anxiety, there are several studies that indicate that between $50 \%$ and $60 \%$ of marijuana users have suffered between one and more episodes of anxiety

When consumed in groups or in favorable social environments, the predominant symptoms are greater euphoria, which is expressed as hilarity with unmotivated laughter, logorrhea, and gregariousness

When consumption is individual, the dominant symptoms correspond to the effects of pressure: drowsiness and apathy.

It causes disturbances in the subjective perception of time (minutes seem like hours). It can produce visual hallucinations auditory and tactile. In general, acute intoxication usually predominates, at the end of the episode: sedation, drowsiness and lethargy. Disorders of concentration and recent memory appear.

Between the effects they stand out: alteration of the motor coordination, tachycardia, orthostatic hypotension, increase of the appetite, mydriasis, and conjunctival injection.

It also causes transient palpebral ptosis, amimia, dry mouth and inhibition of sweating.

In summary, the most important effects occur in the cardiovascular system and in the CNS with the risk of psychosis

The "white" functions (that is, the ones that act selectively) are:

I. Motor coordination

II. Cognitive function

There is a commitment to humor, memory, attention and processing of information and reduction of reaction time, even with moderate or low doses

\section{These effects persist $4-8 \mathrm{~h}$. after the subjective effects of the drug have decreased.}

With respect to psychosis, studies in Spain conducted by Ana Gonzáles Pinto ${ }^{9}$ and collaborators, show that immature brains are more vulnerable to trigger psychosis with the use of marijuana. They also show that the brain damage produced by cannabis that is expressed by a first episode of psychosis, can be reversible late (at 5-8years) if the patient abandons consumption. If you do not do it, the evolution is serious. It also concludes that marijuana precipitates this disease in vulnerable people, who without the consumption would not have suffered from the disease. "These studies show that cannabis use represents a risk factor for suffering schizophrenia."

Currently, it is mentioned, although it is not frequent, that a first or only consumption can induce a Transient Toxic Acute Psychosis (PATT), characterized by the presence of paranoid hallucinations with intact reality judgment. The risk of suffering a permanent psychotic disorder is 2 to 3 times higher in those who are frequent consumers. ${ }^{1}$ Other researchers have confirmed the above, recognizing the effect in the psychiatric sphere, the main toxicity of marijuana. Anxiety is observed in 20 to $25 \%$ of consumers, being more frequent in oral consumption because doses cannot be regulated as in the inhalation route. In this way, this symptom manifests itself predominantly in non-experienced people. ${ }^{1}$ In Goodman and Gilman, ${ }^{24}$ it is mentioned that diverse investigations show that anxiety is a more frequent 
symptom than suspected, reaching $50-60 \%$ of consumers who have suffered it less once.

\section{Also the bibliography states that anxiety can lead to the appearance of anguish crisis}

Recently different studies in the exposed population show that the consumption of this substance is associated with a 2.5 times higher risk of activating the bipolarity genes and this risk increases the earlier the age is. These genes are not present in the whole population, but in especially vulnerable people, they can be activated in a first consumption. The average age of those affected by this bipolar disorder is around 30years, although one in two cases have its origin in adolescence. ${ }^{30}$

Genetic vulnerability: It has now been scientifically proven that there is a relationship between marijuana, psychotic disorders and individual consumer vulnerability. Among these different vulnerabilities, genetic predisposition acquires special importance. This consists, according to the researches proven so far, that patients who have a specific variation of the AKT1 gene, which programs the enzyme that affects the signaling of dopamine in the striatum, are more prone to develop psychosis. In these cases, people who consume marijuana daily have a risk seven times greater than those who use it infrequently or do not consume it.

Although psychosis can manifest itself in adulthood, the use of marijuana during adolescence in a population with genetic predisposition represents the potential risk mentioned above. ${ }^{5}$ In another earlier study increased risk of psychosis in adults who had used marijuana during adolescence and had a specific variant in the gene catechol O-methyltransferase (COMT) was discovered. This is an enzyme whose function is to degrade a whole class of neurotransmitters including dopamine and norepinephrine and other brain chemicals involved in schizophrenia. This gene has two varieties: Met and Val. Only the Val variety is involved in the disease, even if they are one or two copies. The exclusively Met variant has no connection. ${ }^{4}$

It has also been recognized that when there is a concomitant abuse of alcohol and/or drugs, patients with bipolar disorder have "more persistent, severe and resistant to treatment" symptoms. ${ }^{25}$ According to the above expert, these patients are estimated to commit suicide by 15 to $20 \%$ of cases.

In conclusion, it is recognized that there is sufficient evidence that the use of marijuana, especially if it is intense and of early onset (adolescence), in which the CNS is still in the process of maturation, can produce prolonged and/or permanent neuropsychological damage. In patients with arterial hypertension, cardiovascular diseases and/ or coronary heart disease, the increase in cardiac work produced by cannabinoids represents a potential risk. With habitual, intense and chronic consumption, respiratory toxicity is manifested through the inhalation of smoke. It is expressed by a higher frequency of cough, bronchitis, dyspnea, neuropathies, asthma, and emphysema. It also adds its potential carcinogenic effect.

These manifestations are also related to the technique of smoking marijuana in which the inspirations tend to be longer and deeper than in the consumption of tobacco, in order to intensify its effect. Smoking associated with cannabis use should also be considered, and the installation of Chronic Obstructive Pulmonary Disease (COPD) should be encouraged. It is not frequent that consultations are carried out in Emergency Services of Assistance Centers for acute poisonings due to marijuana use. These consultations are usually produced by patients who perform their first experimentation, often accompanied by their peer group. Experienced smokers usually self-monitor doses, trying to avoid the need for medical consultation. At low doses, the motor effects consist of a decrease in muscle strength, an alteration in the balance that is accentuated by the increase in doses. Cardiovascular effects are mainly tachycardia ( 30 to 60 beats/minute above the values present before smoking) and increased cardiac output and orthostatic hypotension.

These effects are attenuated with both propranolol and atropine, which shows the sympathetic and parasympathetic influence of the Autonomic Nervous System. The initial cutaneous vasoconstriction, mediated by alpha receptors, progresses with high doses towards peripheral vasodilatation and deterioration of sympathetic vascular reflexes, causing orthostatic hypotension. In chronic consumers, orthostatic hypotension is attenuated by a gradually increasing volume blood the $\Delta^{9}$-THC, smoked as administered intravenously, has an important bronchodilator effect, both in normal people and in asthmatics, but its habitual use leads to the appearance of chronic obstructive pulmonary disease (COPD).

It produces an increase in sensitivity to external stimuli, colors are perceived brighter and brighter and there is a subjective feeling that time passes more slowly ("minutes seem hours"). The toxic reactions produced by high doses rally consist of delirium, mutism and/or acute cerebral organic syndrome. Motor coordination disorders still appear at low doses and their duration is longer than the effects of euphoria. They may persist for more than 12 hours. Movement disorders are also associated with accidents. This risk is accentuated if there is alcohol consumption, even with low doses of both drugs due to the potentiation of their effects. It has also been shown that the use of tobacco and marijuana makes it difficult to abandon the former.

The intravenous injection of a boiled marijuana extract caused a disease characterized by: headache, blurred vision, sweating, chills, and dizziness, dyspnea, followed in the following hours of hyperthermia, myalgia and severe diarrhea.

It has also been reported a childhood intoxication by ingesting cannabis or resin cookies causing hypothermia, hypotonia, ataxia, affective liability, vertical or horizontal nystagmus, tremors, tachycardia/bradycardia, conjunctival injection and pallor, stupor and lethargy that progress rapidly to coma that can last up to 36-40hours.

The $\Delta^{9}$-THC can be detected in urine between 3 and 8 weeks in chronic consumers and between 3 and 4 days in sporadic consumers, depending also on the sensitivity of the method. It must be borne in mind that dilution of the sample, the addition of some adulterants such as detergents, salt, vinegar or the use of diuretics can cause a falsenegative result of the sample. There may also be false positives when the following drugs are present in the urine: ibuprofen, fenoprofen or naproxen.

\section{Treatment of acute intoxication}

The poisoning does not have specific treatment. It must be implemented according to the case:

a. Psychological containment measures

b. Psychological containment measures plus general support measures

c. Psychiatric patients may require, according to type and magnitude 
Benzodiazepines (Diazepam or Lorazepam) for moderate anxiety or panic:

Diazepam: IV slow bolus 5-10mg. Maximum dose 40mg.

Lorazepam: Vía IV or IM in deltoids if there is no access to IV: $2-4 \mathrm{mg}$.

Midazolam: Vía IV in slow bolus 5-10mg. It can be repeated.

Generally only general supportive measures and psychological support is required, the latter if the patient's condition permits. Monitor orthostatic arterial hypotension. In cases of severe poisoning in adolescents, an inpatient stay of up to 24hours may be necessary or more depending on the severity of the complications. If necessary the minimum dose Useful (DMU) benzodiazepine be used as marijuana produces multiple effects sobe CNS and psychomotor excitement euphoria, hallucinations and likely decreased awareness; being these symptoms those that dominate in the last clinical stage of the intoxication.

\section{Chronic intoxication}

Prolonged use of marijuana causes changes in hippocampal neurons, indicating that there may be negative effects on personality. Also, marijuana smoke contains $50 \%$ to $70 \%$ more tar, from the pyrrolysis of carcinogenic aromatic hydrocarbons, than that of tobacco. Another risk factor is the technique of smoking, (deeper inhalation and retention of inhaled air longer than smokers snuff). It has also been observed that consumers under the age of 15 have greater health problems: lung diseases, visual disorders and depressive disorders.

With respect to schizophrenia. As a summary it is mentioned that it has been detected in exposed populations that there is an incidence up to 6times higher than in non-exposed populations. There is also a positive correlation between concentration of $\Delta^{9}$-THC and psychosis (the higher the concentration of the active substance, the greater the risk of suffering from this pathology.

Chronic consumers are also more susceptible to psychiatric crises. In them, the appearance of paranoid ideas is more frequent than psychosis itself. In conclusion, it is recognized that there is sufficient evidence that the consumption of Cannabis, especially if it is intense and starts at an early age, has a higher risk of producing disorders neuropsychiatric prolonged and/or possibly definitive

Other observable manifestations with chronic consumption are:

1. Chronic fatigue

2. Lethargy

3. Headaches

4. Irritability

5. Disinhibition

6. Discoloration of tongue

7. Uvula edema

8. Dry cough

9. Chronic ulcer of the isthmus of the fauces

10. Nasal congestion

11. Exacerbation of asthma

12. Frequent infections of the respiratory tract
13. Chronic bronchitis

14. Chronic obstructive pulmonary disease (COPD)

15. Disorders of immunity (decreased activity of $\mathrm{T}$ and $\mathrm{B}$ lymphocytes)

16. Lung cancer

17. Decreased motor coordination

18. Increased reaction time

19. Commitment of the visual field

20. Alterations of color and depth of visual perceptions

21. Amenorrhea or dysmenorrhea (menstrual disorders)

22. Probable Infertility

23. Impotence

24. Decreased libido

25. Teratological abnormalities.

26. Mood disorders (depression, anxiety)

27. Panic attacks

28. Suicide attempts

29. Changes in personality

30. Decreased REM sleep (rapid eye movements)

31. Alterations of memory (amnestic gaps)

32. Commitment to abstract reasoning

33. Changes or disorders in the behavioral sphere

34. Delirium

35. Psychosis

36. Flash-back

The presence of abnormal sperm with decreased motility has been observed. Also decrease of plasma testosterone, gynecomastia and reduction of testicular size. There is evidence that marijuana causes decreased immunity with decreased levels of $\mathrm{T}$ and $\mathrm{B}$ lymphocytes. It should be considered, in the evaluation of the risks, that the smoke of marijuana, although it does not contain nicotine, contains carbon monoxide, acetaldehyde, acrolein, toluene, phenol, cresol, cyanide, acetone and ammonia, among other toxic agents. In its composition involved substances 10times more irritating to the respiratory tree and carcinogenic than in tobacco, as well as benzene, benzoanthracene and benzopyrene in amounts greater than twice those found in tobacco smoke.

There are studies that relate the prolonged use of the drug with cerebral atrophy and greater susceptibility to suffer psychiatric crisis. In them, the appearance of paranoid ideas is more frequent than psychosis itself. These manifestations are usually associated with previous personality disorders. Studies of neuroimaging have described unique cases of cerebral infarctions, their mechanism of action is unknown and if the cause-effect relationship is certain. In conclusion, to date the results are inconclusive. This is due to the fact that some studies describe the reduction of gray matter parahipocampica and also certain changes in the hippocampus, but they are not consistent in all the works. 


\section{Syndrome amotivational}

It is characterized by apathy and disinterest in activities that are rewarding and in achieving goals. The impact of the drug on motivation and performance varies according to:

a) The personality characteristics

b) The characteristics of the medium

Controversies exist if this syndrome is produced specifically by marijuana or if it responds to pre-existing psychopathological characteristics. At present it is thought that it can be produced also by other drugs and therefore it would be one of the characteristics of the drug-dependent patient. Alterations in the performance of complex tasks could also be related to alterations in concentration, short-term memory compromise and decreased energy levels caused by the residual effect of marijuana.

Flashback: ("Persistent Perceptual Disorder by Hallucinogens"). They are usually short events (from seconds to hours) that are usually triggered by stressful situations, the use of other drugs or environmental stimuli that are eminiscent of the original hallucinatory experience. It can be pleasant or unpleasant and occur spontaneously, weeks or months, after the original experience. The tendency of the flashback is to decrease in intensity, frequency and duration. In patients in whom the experience of the flashback lasts more than a year of the original experience, although cases of up to 2years are reported, the presence of psychiatric disorders linked or independent of the addiction must be considered. The Cannabis can exacerbate psychotic symptoms in patients with major psychotic disorders, such as schizophrenia. In psychotic syndromes that last beyond a week after cessation of cannabis use should be considered highly probable the existence of a premorbid predisposition to psychosis, independent of $\Delta^{9}$-THC, although this has acted as trigger.

\section{Abstinence syndrome:}

It happens even after brief periods of use like 21days.

The symptoms are:
a. Yawns
b. Irritability
c. Restlessness
d. Excitement
e. Anxiety consumption)
g. Anorexy
h. Depression
i. Thinning
j. Insomnia
k. Sickness
1. Myalgia
m. Increased REM sleep rebound
n. Tremors
o. Hyperthermia, and chills

f. Aggressiveness (more accentuated to the week of the last
p. Diarrhea
q. Diaphoresis
r. Photophobia

\section{Medical uses}

Its medicinal use is controversial and is controlled by National and International State Organizations, due to the adverse psychotropic effects of THC on the Central Nervous System, particularly psychosis and other psychophysical manifestations. Smoking marijuana should not be considered as an adequate therapeutic behavior for any pathology. These are drugs prepared by the Pharmaceutical Industry for therapeutic purposes.

They have been proposed for the following pathologies, although none of them are considered first line nor used as monodrugs. Nor do they apply in all countries.

Combined treatment of vomiting produced by the chemotherapeutic treatment of cancer. The indication is for the antiemetic effect of marijuana, combined with other drugs.

a. Treatment of Caquectising Syndrome associated with Acquired Immunodeficiency Syndrome (AIDS).

b. It is based on the effect antinauseous and in the increase of the appetite that produces

c. Treatment of Neuropathic Pain of different origin, including that of sclerosis multiple in which it adds its antispastic action to its analgesic properties. ${ }^{1}$

d. Marijuana oil is currently being used (hemp phenotype with high concentration of $\mathrm{CBD}$, for the treatment of refractory epilepsy. It would be indicated in the absence of therapeutic effect anticonvulsant of the usual antiepileptics.

e. Recently also for the treatment of pain caused by some neoplasms

f. The scientific evidence at the preclinical level suggests that cannabinoid agonists of type $\mathrm{CB} 2$, lacking the psychotropic effects of THC, have analgesic properties, relieving osseous oncological pain and decreasing tumor growth, improving the quality of life of affected patients of this pathology.

g. Several studies of animal experimentation have shown that CB2 agonists have antitumor effects and increase the survival of animals affected by breast cancer.

To date, cannabinoids are not used as first-line monopharmaceuticals for the treatment of cancer, but as palliative adjuvants. ${ }^{10}$ It is added as a possible treatment of ocular hypertension for its property hypotensive. There are two factors of questioning its therapeutic indication:

I. Its Adverse Effects (mentioned below)

II. The lack of its specificity in its action

To date, the best known pharmacological products are:

a. The Dronnabinol. Trade name: "Marinol" ( synthetic form of delta-9-tetrahydrocannabinol (capsules of $2.5 \mathrm{mg}, 5 \mathrm{mg}$ and $10 \mathrm{mg}$ ).It is usually indicated in the treatment of nausea and vomiting caused by cancer chemotherapy, anorexia-cachexia syndrome associated with AIDS, neuropathic pain in multiple sclerosis and other etiologies. 
b. Approved by the FDA in 1986 .

c. The Nabilone (analog of cannabinoids). It is an agonist of CB1 receptors.

d. It is marketed under the trade name "Cesamet" and is also indicated for antiemetic treatment of patients undergoing chemotherapeutic medication for cancer that do not respond to other antiemetic drugs.

e. Currently, in the countries of the Americas, it is available in the legal pharmaceutical market in the US, Canada and Mexico.

f. The Sativex contains Cannabis Sativa Extract with Tetrahydrocannabinol (THC) and Cannabidiol (CBD).

g. THC activates $\mathrm{CB} 1$ and $\mathrm{CB} 2$ receptors. $\mathrm{CBD}$ can potentiate the effects of THC and modulate the TRPVA receptor (vanilloid receptor). It is prescribed in countries that authorize its use for neuropathic pain in Multiple Sclerosis and most recently as an adjunct in the palliative treatment of pain caused by cancer. In the year 2005 it was approved in Canada. Presentation: Sublingual and oropharyngeal spray.

h. Cannabis oil: It is the most recent application, which is being used for the treatment of refractory epilepsy.

It is called "Charlotte Oil", because it was used for the first time in California, USA, as a treatment, in a girl with that name, 6 years old, who suffered from a West Syndrome, characterized by suffering more than 300 morning seizures, without sufficient response to conventional anticonvulsant medications.

The patient was a carrier of a SCN1A genetic mutation that is found in $80 \%$ of cases with DRAVET syndrome (Severe Myoclonic Epilepsy of Childhood (SMEI), which is characterized by an undetermined location, as well as generalized and focal seizures. It would also be indicated in other infantile epileptic encephalopathies such as LENNOX-GUSTAUT Syndrome and WEST Syndrome. The cannabis strain used is low in THC and high in CBD, two of the active components of cannabis. Remember that THC is the main psychoactive component of the plant (SEE ALSO FORMULA OF THE PHENOTYPE). It is thought that CBD would decrease the chemical and electrical activity of the brain that causes seizures, having sedative properties. It was called "Cannabis. Charlott's Web strain "to this purified extract of CBD. The information obtained expresses the strain used has $0,5 \%$ of THC and CBD $17 \%$, decreasing seizures in a 50 to $90 \%$.

This CBD oil must be differentiated from "Rick Simpson oil", in which the concentrations of THC (50-60\%) are high and of other cannabinoids (CBN and CBG), in relation to CBD (10-15\%), considering as a total value all cannabinoids (100\%). This would correspond to the "drug" phenotype strain. If one considers the total composition of Charlott's oil, including all its components, THC represents only $0.173 \%$ and $\mathrm{CBD}$ represents $4 \%$. Both oils (Charlott e and Rick Simpson) are different strains and therefore their effects too. It seems only mentioned for the first of them, the risk of producing digestive disorders. In Argentina, the ANMAT (National Administration of Drugs, Food and Technology)-INAL (National Food Institute), authorized the importation of USA, of HEMP EXTRACT IN MCT as a compassionate use product for a girl with encephalopathy (West Syndrome) with date 16-10- 2015.
The Province of Chubut is the first province to include Cannabis Oil in the Provincial Health System, by incorporating it into the Vademecum for the treatment of "Dravet Syndrome", better known as epilepsy and other pathologies that the Ministry of Health of the Province, consider it convenient (Law 588 of 09-23-2016). Previously, the Legislature of Chubut had approved it (August 2016). Later, after Chubut, the provinces of Neuquén, Santa Fe, Salta and Mendoza, by provincial laws enabled the medicinal use of cannabis oil. At the national level, the Congress authorized by Law dated 03-23-2017, the therapeutic and palliative use of cannabis and its derivatives, such as Cannabis Oil, with the State being responsible for the monopoly of cultivation and the elaboration of the product used for the treatments. This law creates the regime that drives medical and scientific research into the medicinal use of the plant and its derivatives. To this end, it designates the Ministry of Health of the Nation as responsible for guaranteeing the supply of the necessary supplies to patients who require it, allowing importation and production by the State. In this regard, the National Commission for Scientific and Technical Research (CONICET) and the National Institute of Agricultural Technology (INTA) are authorized to grow the plant. The law also enables the National Administration of Medicines, Food and Medical Technology (ANMAT) to allow the importation of cannabis oil whose provision will be free for patients registered in the National Program that creates the law.

Recently, the Province of Jujuy, by order of the governor, began cultivation for therapeutic purposes. Garrahan Pediatrics Hospital, with national authorization, through a multicenter study, uses oil produced and donated by a Canadian company, currently 21 patients enrolled, but the goal is to reach 100, of which 50 will be from the interior of the country. The doctors have good expectations. Children included have epileptic encephalopathies refractory to drug treatment and other therapies. It is estimated that between $20 \%$ and $30 \%$ of epilepsies are resistant to classical treatment. In the study that will last for 2years, conventional treatment is not withdrawn, but cannabis oil is added in a dose dependent on weight:

In less than 45 kilos begins with 2, 5mg/kilo per day. In children with greater weight they are indicated $5 \mathrm{mg} /$ kilo per day. According to the response, the dose is adjusted up to a maximum of $25 \mathrm{mg} /$ day divided into 2 doses. The study of cannabis for therapeutic purposes is progressing. In addition to its use in epilepsy, research on its virtues (relief of pain), reduction of inflammation, stimulation of appetite, reduction of vomiting and nausea among others. It is expected that in March of this year, WHO will release a definitive review of the scientific literature on this topic. Last year, the US Food and Drug Administration (FDA) approved the first drug containing cannabidiol (CBD), one of the active ingredients in cannabis sativa. In fact, it is not yet known how he acts, the works carried out show empirical evidence. In summary, it is considered important the deepening of studies of Cannabinnoides CBD with certain therapeutic purposes, currently as an alternative medication, when conventional treatment is not sufficient or adequate.

\section{Adverse effects}

\section{Dronnabinol:>10\%}

A predominance of CNS: Sedation (53\%). Drowsiness (48\%). Confusion (30\%) Vertigo, Fainting (21\%). Anxiety. Humor changes. Deficit of concentration Gastrointestinal: Increased appetite (may be a difficulty when used as an antiemetic). Xerostomia (38 to 50\%). 


\section{I to $10 \%$ :}

Cardiovascular: Orthostatic hypotension. Tachycardia.

CNS: Ataxia (4\%). Sensorial depression (7\%). Headaches, vertigo, hallucinations $(5 \%)$. Lapsus in memory $(4 \%)$.

Neuromuscular and skeletal: Paresthesias and weakness. $<1 \%$ :

Limited exclusively to important manifestations or with potential risk to life: Diaphoresis. Diarrhea. Myalgia Nightmares. Syncope Tinnitus of Lacy, Charles F, et al. ${ }^{14}$ in "Drug Information Handbook with INTERNATIONAL INDEX. LEXI-COM'S.USA, 2003.

Due to its important central sympathomimetic activity can produce palpitations, tachycardia, vasodilatation and secondary arterial hypotension, conjunctival hyperemia, excitement similar to that of marijuana, anxiety, nervousness, euphoria, drowsiness, indifference, dizziness, panic, paranoid reactions and cognitive alterations. Sometimes it can cause Syndrome of Abstinence due to sudden suppression: irritability, insomnia, and restlessness.

Precautions: The Patients with a history of alcohol and other drug use may consume excessive doses.

\section{Nabilona:}

Adverse effects similar to those of Dronnabinol with important effects on the CNS in> $10 \%$ of patients. ${ }^{27}$

\section{Synthesis of the clinical table of acute intoxication}

Syndrome of acute infantile poisoning 1 by ingestion of marijuana crackers
a. Pallor
b. Hypothermia
c. Ataxia
d. Hypotonia
e. Affective lability
f. Mydriasis/Miosis/Normal pupils
g. Conjunctival injection
h. Vertical or horizontal nystagmus
i. Tremors
j. Involuntary and uncoordinated movements
k. Tachycardia/Bradycardia
1. Stupor
m. Lethargy that progresses rapidly to
n. Eat (lasting up to 40hours)

\section{Acknowledgments}

None.

\section{Conflicts of interest}

Author declares that there are no conflicts of interest.

\section{References}

1. Ayesta FJ, Rodríguez M, Santamaría, J. Cannabis Therapeutic utility in Disorders Addictive/Drug dependence. In: Flórez Jesús, Armijo, Juan A, Mediavilla, Africa. Human Pharmacology. $6^{\text {th }}$ edition. Elsevier Masson, Barcelona: Spain; 2014.

2. Benjamin, Marny Marijuana (THC) in Harris, Carson R. Manual of Toxicology for Physicians. Elsevier Masson, Barcelona; Spain. 2008.

3. Calafat Far A. Cannabis: Clinic in SET Treaty of Disorders Addictive. Perez Directors of the Cobos JC; Valderrama Zurián JC; Cervera Martínez G; Rubio Valladolid G. Spanish Society of Drug Addiction. Appointment Set edition. Panamericana Medical, Madrid: Spain; 2006.

4. Caspi A, Moffitt TE, Cannon M, et al. Moderation of the effect of adolescent-onset cannabis use on adult psychosis by a functional polymorphism in the catechol-O-methyltransferase gene: longitudinal evidence of a gene $\mathrm{X}$ environment interaction. Biol Psychiatry. 2005;57(10):1117-1127.

5. Di Forti M, Iyegbe C, Sallis H, et al. Confirmation that the AKT1 (rs2494732) genotype influences the risk of psychosis in cannabis users. Biol Psychiatry. 2012;72(10):811-816.

6. Fernández Ruiz J, Lorenzo Fernández P, Leza Cerro JC. Cannabis (1) Pharmacology in Drug Addiction. Lorenzo Fernández P, Ladero JM, Leza JCy Lizasoain I. $3^{\text {rd }}$ edition. Editorial Panamericana Medical. Madrid, Spain; 2009.

7. Fernández Ruiz J, Ramos Maple. Cannabis, Biological Bases. In: Pérez de los Cobos Peris, José C, Valderrama Zurián JC, Cervera Martínez G, Rubio Valladolid G. SET Treaty of Addictive Disorders. Spanish Society of Drug Addiction. Editorial Panamericana Medical. Madrid; 2006.

8. Flórez Jesús. Coadjuvant Drugs in the Treatment of Pain. In: Flórez, Jesus. The Pharmacological Treatment of Pain. Ars Medical Barcelona. Spain; 2007.

9. Gonzales Pinto Ana. Marijuana Drugs Info. Spain; 2010.

10. Guillén MR, Jiménez JM. Breast Cancer and Cannabinoids. In: Flores, Juan Carlos. Pain Medicine. International Perspective Elsevier. Barcelona, Spain; 2015.

11. Llanero Luque, Pedrero Pérez, Ruiz Sánchez de León, et al. Neuroimaging at Study of Addiction in Ruiz Sánchez from Lion. In: Jose M, Pedrero Pérez, Eduardo. J Neuropsychology of The addiction. SET ed. Medica Panamericana Madrid. Spain; 2014.

12. Harris Carson R. Cannabinoids. In: Erickson, Timothy B, Ahrens, William R. Aks, Steven AND, Baum, Carl R Ling, Louis J. Pediatric Toxicology. McGraw-Hill Co. USES; 2005.

13. Europe Press. Cannabis use can awaken the genes of bipolar disorder. The drug info. Spain; 2016.

14. Lacy Charles F, Armstrong Lora, Goldman Morton, et al. Dronnabino 1. In: Drug Information Handbook, $11^{\text {th }}$ ed. International Index. USA; 2003.

15. Lacy Charles F, Armstrong Lora, Goldman Morton, et al. Drone aby 1. In: Manual of Medical Prescription $19^{\text {th }}$ ed. American Pharmacists Association. USA; 2011.

16. Leikin Jerrold B, Paloucek Frank P. Marijuana (C annabis). In: Poisoning \& Toxicology Handbook. $3^{\text {rd }}$ ed. USA; 2002.

17. L Loret, J. Derivatives of Cannabis. In: Net Álvar and Morocco-Sant: Poisoning Acute Graves. Ars Medical Barcelona. España; 2006.

18. Mc Guillan, Michael A. Cannabinoids. In: Goldfrank's Emergencies Nelson LS. Lewin NA, Howland MA, Hoffman RS, Goldfrank L Flomenbaum, NE $9^{\text {th }}$ ed. Mc Graw-Hill Medical: USA; 2011. 
19. M arquez López Mato, Andrea, Vallejo, et al. Neurotransmission. In: Vallejo, Norma Elena and Col, Abuse drugs. Toxicological aspect: Symptoms, Diagnosis and Treatment. $1^{\text {st }} \mathrm{ed}$. Argentina.

20. Murray Lindsay, Little Mark, Pascu Ovidiu, et al. Cannabinoids (Marijuana). In: Toxicology Handbook $3^{\text {rd }}$ ed. Elsevier Australia. 2015.

21. National Institute of Drugs Abuse (NIDA). Marijuana. 2005

22. National Institute of Drugs Abuse (NIDA) Advancing Addiction Science. The Marijuana? There is any connection between the use of marijuana and mental illness? 2015.

23. Núñez Domínguez, Luis Alfonso. Cannabis and PsychiatricPathology: An Update. The Journal of Applied Research. 2004;4(1):164-172.

24. O'Brien Charles. Addiction and Drug Abuse: Cannabinoids in Goodman \& Gilman. The bases Pharmacological of Therapeutics. Brunton, Laurence, editors. Associates Chabner, Bruce \& Knollman, Björn. McGraw-Hill: USA; 2012.

25. Popovic Dina. Bipolar disorder, the disease of emotions, in the $21^{\text {st }}$ century. 2016.
26. Ruiz Sánchez de León, José M, Pedrero Pérez Eduardoy Col. Neuropsychology of Addiction. Panamericana Medical. Spain; 2014.

27. Sharkey Keith A, Wallace John L. Treatment of intestinal motility disorders and water flow. Antiemetics in Goodman and Gilman: The Pharmacological Bases of Therapeutics. Laurence L Brunton, et al. 12 ed. Mc Graw Hill: Mexico; 2012.

28. Vallejo Norma Elena. Marihuana. In: Vallejo Norma Elena Col. Aspectos Toxicológicos of the Drogadependencia. $3^{\text {rd }} \mathrm{ed}$. Sedronar, Buenos Aires. Argentina; 2011.

29. Vallejo Norma Elena. Marihuana. In: Abuse Drugs, Toxicological Aspects: Symptoms, Diagnosis and Treatment. Akadia, editors. $1^{\text {st }}$ ed. Buenos Aires, Argentina; 2019.

30. Vieta Eduard. International Symposium Bipolar disorder, the disease of emotions. In: XXI Century. 2016. 